\title{
3D Nanostructured Palladium with Single Diamond Architecture for Enhanced Catalytic Activity
}

\author{
Matthew Burton ${ }^{\dagger}$, Anand Selvam ${ }^{\dagger}$, Jake Lawrie-Ashton ${ }^{\dagger}$, Adam Squires ${ }^{\ddagger}$, Nicholas Terrill ${ }^{\S}$, Iris Nandha- \\ $\operatorname{kumar}^{\dagger} *$ \\ ${ }^{\dagger}$ Department of Chemistry, University of Southampton, University Road, Highfield, Southampton, SO17 1BJ, United King- \\ dom \\ ${ }^{\ddagger}$ Department of Chemistry, University of Bath, Claverton Down, Bath, BA2 7AY, United Kingdom \\ ${ }^{\S}$ Diamond Light Source, Diamond House, Harwell Science and Innovation Campus, Didcot, Oxford, OX11 0DE, United \\ Kingdom \\ *E-mail: iris@soton.ac.uk
}

\begin{abstract}
Fuel cells are a key new green technology that have applications in both transport and portable power generation. Carbon supported platinum (Pt) is used as an anode and cathode electrocatalyst in low-temperature fuel cells fuelled with hydrogen or low molecular weight alcohols. The cost of Pt and the limited world supply are significant barriers to the widespread use of these types of fuel cells. Comparatively palladium has a three times higher abundance in the Earth's crust. Here a facile, low temperature and scalable synthetic route towards 3D nanostructured palladium (Pd) employing electrochemical templating from inverse lyotropic lipid phases is presented. The obtained single diamond morphology Pd nanostructures exhibited excellent catalytic activity and stability towards methanol, ethanol and glycerol oxidation compared to commercial Pd black and the nanostructure was verified by small-angle X-ray scattering (SAXS), scanning tunneling electron microscopy (STEM) as well as by cyclic voltammetry (CV).
\end{abstract}

\section{INTRODUCTION}

Nanostructured palladium (Pd) has applications in gas sensors, ${ }^{1,2}$ electrochemical biosensors, ${ }^{3}$ and supercapacitors. ${ }^{4,5}$ However, one of the largest areas of interest are fuel cells which are seen as a key green technology with applications in transport and portable power generation. Currently fuel cells are electrocatalysed by platinum (Pt) based materials, but the cost and limited supply of Pt makes this option far from ideal. Comparatively palladium (Pd) has a three times higher abundance in the Earth's crust at $0.015 \mathrm{ppm}$ by weight compared to Pt at 0.005 ppm. ${ }^{6}$ Pd is similar in price to $\mathrm{Pt}$, \$906 per ounce and \$814 per ounce respectively (Kitco Gold Index, Aug 02, 2018), which is still higher than acceptable for commercial applications. However, as a nanomaterial, the levels of Pd required can be lowered, making its use more appealing whilst simultaneously increasing its catalytic activity.

Pd based nanomaterials have been used as catalytic layers for direct alcohol fuel cells (DAFCs) and have been fabricated by many techniques including sputtering, ${ }^{7-10}$ ion or electron beam deposition, ${ }^{11,12}$ and laser ablation. ${ }^{13,14}$ Hydrothermal techniques, ${ }^{15-17}$ and chemical reactions have also been used for palladium nanomaterial formation. ${ }^{18-22}$ Chen et al. summarizes these techniques. ${ }^{6}$ Another technique that can be used is electrodeposition, which is a low-cost, mature, scalable industrial technique compatible with high throughput continuous manufacturing processes. ${ }^{23,24}$

Template-free synthesis of Pd nanomaterials has been achieved electrochemically by cyclic voltammetry that produces nanomaterials with thicknesses of up to around 150 $n \mathrm{~nm} .^{25-29}$ The technique often relies on another element such as
$\mathrm{Pt}^{26}$ and the depositions form non-periodic nanoarchitectures, ${ }^{29}$ nanocomposites, ${ }^{28}$ or nanoparticles, ${ }^{27}$ but have not resulted in periodic nanostructures. Other electrochemical methods for template-free synthesis of Pd nanomaterials include square wave voltammetry, ${ }^{30,31}$ chronoamperometry, ${ }^{32-36}$ chronopotentiometry, ${ }^{37,38}$ chronocoulometry, ${ }^{39}$ and pulse deposition. ${ }^{40}$ The produced Pd nanomaterials largely displayed increased roughness with partial coverage of the substrate electrode and were not ordered.

Template-assisted growth of ordered Pd nanomaterials has been achieved by electrodeposition utilising mostly anodised aluminium oxide (AAO) or porous anodic alumina (PAA) as templates. ${ }^{41-45}$ Wang et al. successfully electrodeposited Pd in AAO producing a nanowire array with an average diameter of $80 \mathrm{~nm} .{ }^{41}$ These nanowires were shown to have a 3 fold increase in the electrooxidation of ethanol by cyclic voltammetry studies compared to non-templated Pd. Bai et al. electrodeposited Pd into CdS modified PAA to produce Pd nanotubes with an average diameter of $200 \mathrm{~nm} .{ }^{45}$ The tubes were used as part of a nonenzymatic glucose sensor, so the effectiveness for a fuel cell was not tested.

Bartlett et al. have used Type 1 normal topology lyotropic liquid crystal phases of non-ionic surfactants such as $\mathrm{C}_{16} \mathrm{EO}_{8}$ and Brij ${ }^{\circledR} 56$ as a template to fabricate hexagonally nanostructured Pd films by electrodeposition. ${ }^{46}$ The pore diameters and wall thicknesses were about $3 \mathrm{~nm}$ each, whilst the pore center to pore center distance was measured to be $5.8 \mathrm{~nm}$. However in this study no fuel cell related electrooxidation experiments were conducted.

We herein report the first successful fabrication of ordered Pd nanomaterials with a single diamond architecture $\left(Q_{227}\right)$ from 
Type 2 inverse bicontinuous cubic lyotropic liquid crystal phases $\left(Q_{224}\right)$ of phytantriol. ${ }^{47}$ The presence of a nanostructure was verified by Small-angle X-ray diffraction (SAXS) and transmission electron microscopy (TEM) and its catalytic activity tested against non-templated Pd and commercial Pd black for the oxidation reaction of methanol, ethanol and glycerol.

\section{EXPERIMENTAL SECTION}

Chemicals and Materials: The Pd precursor electrolyte was prepared by dissolving (NH4) ${ }_{2} \mathrm{PdCl}_{4}$ (Sigma-Aldrich, $\geq 99.995 \%$ ), $\mathrm{NH}_{4} \mathrm{Cl}$ (Fisher, $\geq 99.5 \%$ ) and $\mathrm{HCl}$ (Fisher, ACS plus) in deionised water (Milli-Q 18.2 M $\Omega$ ) to yield concentrations of $50 \mathrm{mM}, 1 \mathrm{M}$ and $1 \mathrm{M}$ respectively. The electrolyte was deaerated by gently bubbling the electrolyte through with argon for 20 minutes prior to use. To perform cyclic voltammetry (CV) of the Pd precursor electrolyte and surface area characterisation a $2 \mathrm{~mm}$ gold electrode encapsulated in Kel-F support (CH101, CH Instruments, Inc.) was used as the working electrode, whilst to test catalytic activity a $3 \mathrm{~mm}$ glassy carbon electrode encapsulated in glass was used. For SAXS studies a Au DVD working electrode was used and for SEM, EDX and XRD a Au on Si wafer was used, both as detailed previously. ${ }^{48,49}$ A deposition potential of $+0.1 \mathrm{~V}$ vs. SCE was used for all Pd deposition and unless otherwise stated deposition was halted once $14.4 \mathrm{mC}$ of charge was passed. For catalytic comparison $38.45 \mathrm{mg}$ of Pd black was applied onto a $3 \mathrm{~mm}$ polished carbon disc electrode by pipetting on $5 \mu \mathrm{L}$ of an ink consisting of $80 \mathrm{mg}$ Pd black (Alfa Aesar, 99.9\%), $40 \mu \mathrm{L}$ Nafion $® 117$ solution (Aldrich) and $1000 \mu \mathrm{L}$ deionized water and leaving in an oven at $100{ }^{\circ} \mathrm{C}$ for at least 15 minutes. Currents were normalized by dividing by the mass of Pd on the electrode. Nanostructured Pd films were prepared by electrodeposition through thin films of phytantriol. 3,7,11,15-tetramethyl-1,2,3-hexadecanetriol, denoted phytantriol, was purchased from DSM Nutrional Products Europe Ltd. The lipid template was applied by dip coating the working electrode into a solution of ethanol and phytantriol 1:2 (w/w). Once dip coated the substrate was left for no less than 30 minutes to allow the ethanol to evaporate, leaving an estimated $22 \pm 1 \mu \mathrm{m}$ thin film of phytantriol coating. ${ }^{50}$ The working electrode could then be immersed into the electrolyte and left to equilibrate for no less than 60 minutes prior to deposition. After electrodeposition the template was removed by rinsing thoroughly in ethanol. To ensure complete removal of the template the films were analysed by EDX and SAXS. The presence of any template left on the substrate surface would have resulted in a prominent carbon EDX signal and additional SAXS peaks.

A saturated calomel electrode (SCE) reference was used to perform CV studies of the electrolyte (Figure S19) and electrochemical deposition. All other electrochemical experiments used a saturated mercury-mercurous sulfate electrode (SMSE) as a reference. Electrooxidation experiments were conducted with $1 \mathrm{M}$ of $\mathrm{KOH}$ and $1 \mathrm{M}$ of the alcohol, with the exception of ethanol where only $0.1 \mathrm{M}$ of ethanol and $0.1 \mathrm{M}$ of $\mathrm{NaOH}$ were used because a lowering of $\mathrm{pH}$ gives conditions that more easily facilitate the complete oxidation of ethanol to $\mathrm{CO}_{2} \cdot{ }^{51,52}$ There were no visible changes to any working electrodes post any characterization or stability measurements. A Pt gauze electrode with area far in excess of the working electrode was used as the counter, which was flame annealed in a blue flame prior to every electrochemical experiment.

Characterization: XRD (Figure S20) was performed on a Rigaku SmartLab with $\mathrm{Cu} \mathrm{K}_{\alpha}$ radiation. SEM (Figure S21) was performed on a Zeiss EVO LS25 ESEM, with an Oxford Instruments energy-dispersive x-ray spectroscopy attachment. Scanning high angle annular dark-field imaging scanning transmission electron microscopy (HAAD STEM) images of Pd directly electrodeposited onto 400 mesh carboncoated Au grids (Agar Scientific) were obtained on a Jeol ARM300CF instrument at the electron Physical Sciences Imaging Centre (ePSIC). SAXS was performed at diamond light source on beam line I22 with a beam energy and size of 12.4 $\mathrm{KeV}$ and $320 \mu \mathrm{m} \times 80 \mu \mathrm{m}$ respectively. A Pilatus $2 \mathrm{M}$ detector was used to collect data over the q range of $0.05 \AA^{-1}-0.30 \AA^{-}$ 1. Calibration was achieved by using a silver behenate sample.

\section{RESULTS AND DISCUSSION}

To identify the presence of a nanostructure of the phytantriol template and the resulting Pd electrodeposits SAXS studies were performed which are shown in Figure 1. Figure $1 \mathrm{a}$ and $\mathrm{b}$ show eight Bragg peaks with relative positions of $1 / \mathrm{d}$ in ratios $\sqrt{2}: \sqrt{3}: \sqrt{4}: \sqrt{6}: \sqrt{8}: \sqrt{9}: \sqrt{10}: \sqrt{ } 11$ which can be indexed as $(\mathrm{hkl})$ = (110), (111), (200), (211), (220), (221), (310) and (311) reflections of a cubic lattice of crystallographic space group Pn3m $\left(\mathrm{Q}_{224}\right){ }^{47}$ This is consistent with previous observations for $\mathrm{Pt}{ }^{53}$ $\mathrm{Bi}_{2} \mathrm{Te}_{3},{ }^{49}$ and $\mathrm{Bi}_{2} \mathrm{~S}_{3} .{ }^{48}$ The derived lattice parameter of the structure is calculated to be $71.0( \pm 0.5) \AA$. 1D SAXS radial profiles and the corresponding 2D SAXS patterns of a Pd film deposited through the $\mathrm{Q}_{224}$ phytantriol structure are shown in Figure 1c and $\mathrm{d}$ respectively. Two Bragg peaks can be seen with relative $1 / \mathrm{d}$ position ratios of $\sqrt{3}: \sqrt{8}$, which can be indexed as the (hkl) $=(111)$ and (220) reflections of the cubic lattice of crystallographic space group Fd3m $\left(Q_{227}\right)$. This is consistent with observations seen for electrodeposited $\mathrm{Pt}$ and $\mathrm{Bi}_{2} \mathrm{~S}_{3}$ through a $\mathrm{Q}_{224}$ phytantriol template. ${ }^{48,50,53,54}$ This observation can be rationalized by electrodeposition occurring preferentially in one of the two water channels of the parent "double diamond" $\left(\mathrm{Q}_{224}\right)$ phytantriol template, resulting in a "single diamond" $\left(\mathrm{Q}_{227}\right) \mathrm{Pd}$ nanostructure. ${ }^{53}$ The lattice parameter of the $\mathrm{Q}_{227} \mathrm{Pd}$ structure is calculated to be $140.0( \pm 4.6) \AA$.

To further confirm the SAXS results seen for electrodeposited Pd through phytantriol, HAAD-STEM was used to image the Pd nanostructure. A selection of resulting images along with their corresponding Fast Fourier Transforms (FFT) can be seen in Figure 2. To aid visualization of the STEM images, MatLab projections of the $\mathrm{Q}_{227}$ structure are also included in Figure 2 . The TEM images show that the Pd nanostructure produced is consistent with MatLab projections of the $\mathrm{Q}_{227}$ structure. 

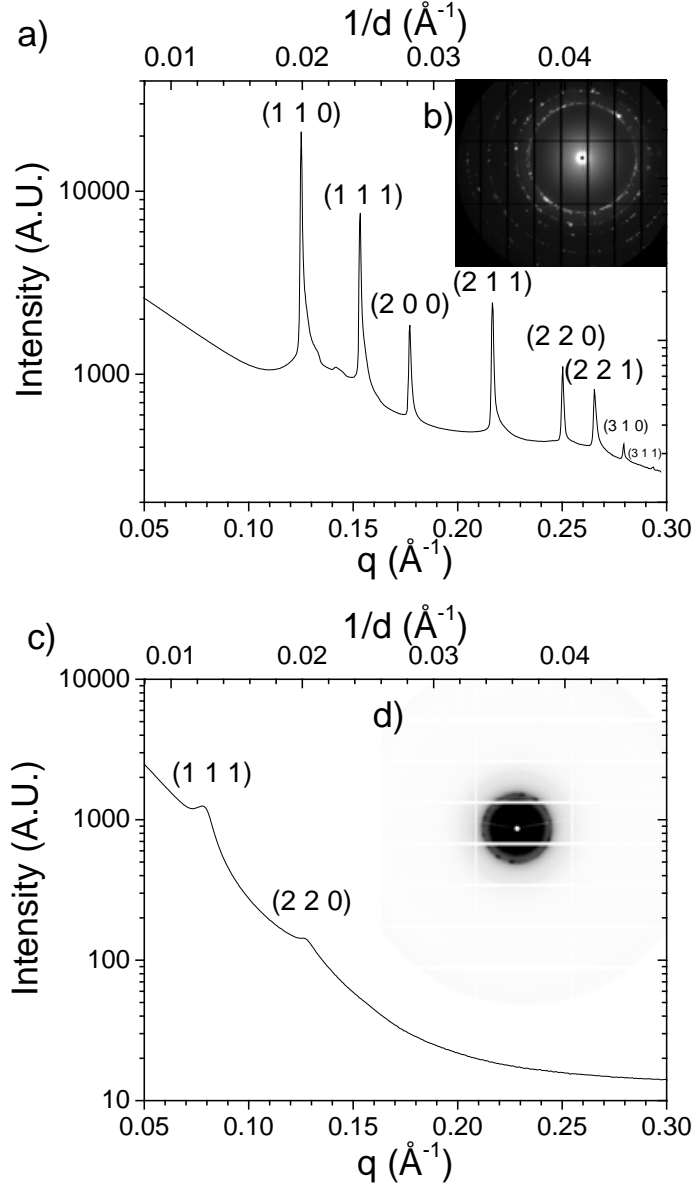

Figure 1. a) 1D integrated SAXS pattern of a phytantriol lined capillary filled with $50 \mathrm{mM}\left(\mathrm{NH}_{4}\right)_{2} \mathrm{PdCl}_{4}$ and $1 \mathrm{M} \mathrm{HN}_{4} \mathrm{Cl}$ in $1 \mathrm{M} \mathrm{HCl}$. b) The corresponding 2D pattern is included in the upper right segment. c) 1D integrated SAXS pattern of a Pd thin film electrodeposited on a phytantriol coated Au DVD. The electrolyte was 50 $\mathrm{mM}(\mathrm{NH} 4) 2 \mathrm{PdCl} 4$ and $1 \mathrm{M} \mathrm{HN} 4 \mathrm{Cl}$ in $1 \mathrm{M} \mathrm{HCl}$ and the deposition conditions were $1800 \mathrm{~s}$ at $+0.1 \mathrm{~V}$ vs SCE. d) The corresponding $2 \mathrm{D}$ pattern is included in the upper right segment.

To further understand the behaviour of the $\mathrm{Q}_{227}$ (single diamond) Pd nanostructure and establish the electroactive surface area, CVs of Pd were run in $1 \mathrm{M} \mathrm{H}_{2} \mathrm{SO}_{4}$ at a scan rate of $20 \mathrm{mV}$ s- 1 . The second scan of a typical CV can be seen in Figure 3a which includes the second scans of CVs of a Pd film electrodeposited in the absence of phytantriol as a planar standard and that of Pd black as a high surface area standard. The first five scans of each can be seen in Figure S1, Figure S2 and Figure S3. For each Pd type hydrogen adsorption can be seen on the cathodic scan from $-0.40 \mathrm{~V}$ vs. SMSE onwards and hydrogen desorption can be seen on the return anodic scan up to $-0.30 \mathrm{~V}$ vs. SMSE. Whilst for non-templated Pd no clear hydrogen adsorption peak is seen, there are distinct hydrogen adsorption peaks for Pd black and single diamond Pd. Pd black has a single hydrogen adsorption peak at $-0.50 \mathrm{~V}$ vs. SMSE (a) that can be seen on the cathodic scan, indicating a single surface environment (which is consistent with a sphere). For $\mathrm{Q}_{227}$ "single diamond" Pd, however, peaks for hydrogen adsorption can be seen at $-0.46 \mathrm{~V}$ vs. SMSE (b), $-0.49 \mathrm{~V}$ vs. SMSE (c) and $-0.53 \mathrm{~V}$ vs. SMSE (d) on the cathodic scan indicating at least 3 different surface environments. Hydrogen absorption can be seen for non-templated Pd, Pd black and single diamond Pd at $-0.65 \mathrm{~V}$ vs. SMSE. Hydrogen desorption peaks can be seen for every hydrogen sorption peak on the anodic scan. The formation of a surface oxide can be seen from $+0.05 \mathrm{~V}$ vs. SMSE on the anodic scan for all 3 Pds, and on the return cathodic scan the stripping of the surface oxide gives rises to peaks that can be seen between $+0.4 \mathrm{~V}$ vs. SMSE and $-0.3 \mathrm{~V}$ vs. SMSE.

Distinctive hydrogen adsorption peaks are a sign of nanostructured $\mathrm{Pd}$, and were also observed for $\mathrm{H}_{1-\mathrm{e}}$ nanostructured Pd by Bartlett et al. ${ }^{46}$ The greatly enhanced peak currents seen for single diamond Pd over non-templated Pd are indicative of a greatly enhanced electroactive surface area.

The surface area was deduced from the charge that was passed by integrated the peak areas under the oxygen stripping peaks. The oxygen striping peaks were integrated between +0.4 $\mathrm{V}$ vs. SMSE and $-0.3 \mathrm{~V}$ vs. SMSE, and a conversion factor of $424 \mu \mathrm{C} \mathrm{cm}^{-2}$ derived by Rands and Woods was used to establish the electroactive surface area. ${ }^{55}$ For a deposition charge of 14.3 $\mathrm{mC}$ for Pd, the surface area was shown to be $0.16457 \mathrm{~cm}^{-2}$ for non-templated Pd, whilst the surface area was shown to be $2.21349 \mathrm{~cm}^{-2}$ for Pd deposited through phytantriol which means a 13.5 fold increase in surface area for the phytantriol templated Pd material. The surface area per gram for non-templated Pd was shown to be $2.11 \mathrm{~m}^{2} \mathrm{~g}^{-1}$, whilst for Pd black the surface area per gram was $17.8 \mathrm{~m}^{2} \mathrm{~g}^{-1}$ (which is expected as the Pd black was described as having a surface area of $20 \mathrm{~m}^{2} \mathrm{~g}^{-1}$ when sold), and for Pd deposited through phytantriol the surface area per gram was shown to be $28.3 \mathrm{~m}^{2} \mathrm{~g}^{-1}$ which is equivalent with a $59 \%$ increase of the surface area per gram of Pd deposited through phytantriol compared to Pd black.

Surface area experiments were conducted for phytantriol templated palladium at several deposition charges between 9 $\mathrm{mC}$ and $50 \mathrm{mC}$. From these experiments roughness factors (Rf) were determined by dividing the electrochemically determined surface area, by the underlying geometric surface area of the substrate electrode. Rf was observed to be linear with deposition charge, as shown in Figure 3b. This linear relationship that passes through the origin, demonstrates a uniformly accessible nanostructure that is not blocked. From the gradient of the plot the specific electrochemical surface area to mass ratio and the surface area to volume ratio were estimated to be $30.8 \pm 0.4 \mathrm{~m}^{2}$ $\mathrm{g}^{-1}$ and $3.66( \pm 0.04) \times 106 \mathrm{~cm}^{2} \mathrm{~cm}^{-3}$ respectively, assuming $100 \%$ faradaic efficiency. Although these are lower than $91 \mathrm{~m}^{2}$ $\mathrm{g}^{-1}$ and $1.1 \times 107 \mathrm{~cm}^{2} \mathrm{~cm}^{-3}$ observed for $\mathrm{H}_{1-\mathrm{e}} \mathrm{Pd}^{46}$ here we show scalability up to at least a deposition charge density of $1.59 \mathrm{C}$ $\mathrm{cm}^{-2}$, whereas Bartlett et al. only used a deposition charge density of $0.89 \mathrm{C} \mathrm{cm}^{-2} .{ }^{46}$ Here we also use substrate electrodes with $4 \times$ the geometric surface area. Therefore in absolute terms we demonstrate the ability to form a single diamond nanostructure using phytantriol which has an accessible Pd surface area over $7 \times$ greater in size than was seen for a $\mathrm{H}_{1-\mathrm{e}} \mathrm{Pd}$ nanostructure created using $\mathrm{C}_{16} \mathrm{EO}_{8}{ }^{46} \mathrm{Pd}$ catalytic activity towards fuel cells is also determined by facets, not simply surface area alone. ${ }^{6}$ This could lead to a $\mathrm{Q}_{227}$ "single diamond" Pd nanostructure having greater catalytic effect per mass than that of $\mathrm{H}_{1-\mathrm{e}} \mathrm{Pd}$ nanostructure.

To assess the potential use of the newly created $\mathrm{Q}_{227} \mathrm{Pd}$ nanostructure in alkaline fuel cells, a series of electrochemical experiments were conducted. From Figure 4a single Diamond 

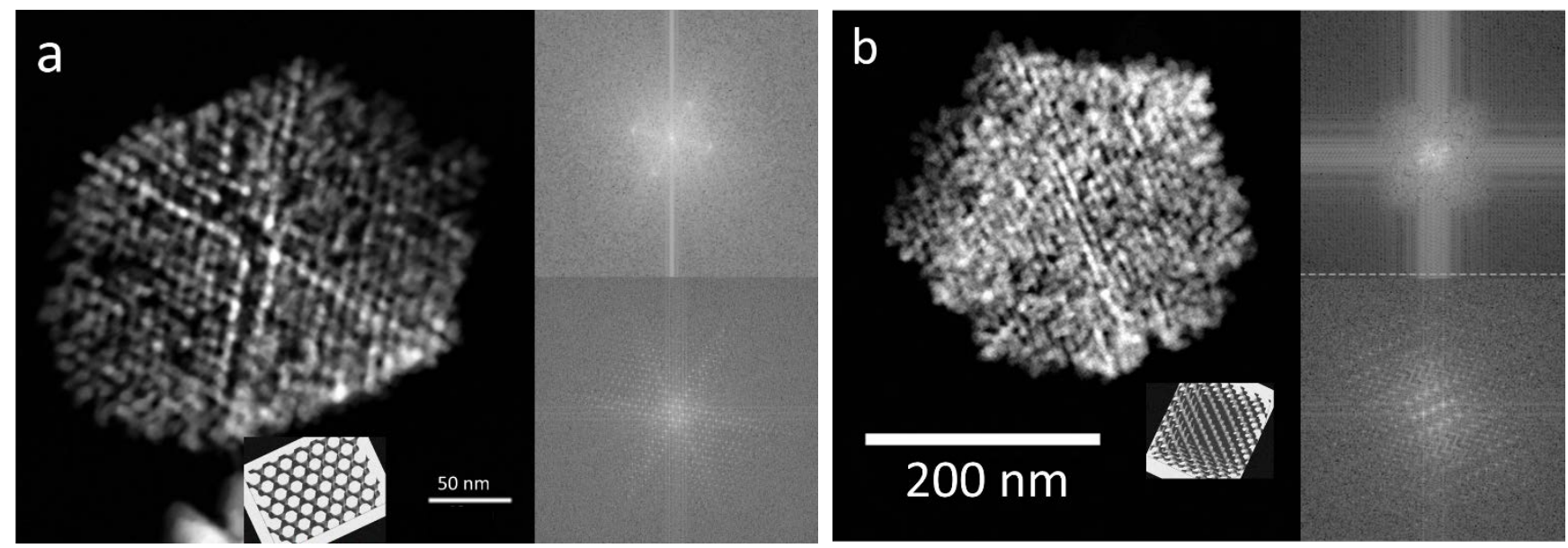

Figure 2. HAAD-STEM images of nanostructured palladium samples and MatLab projections of the Q227 structure with a lattice parameter of $140 \AA$ a) looking along the 110 direction and b) perpendicular to the 111 direction. The top insets show the FFTs of the original STEM images with FFTs of Matlab projections on the bottom.

a)

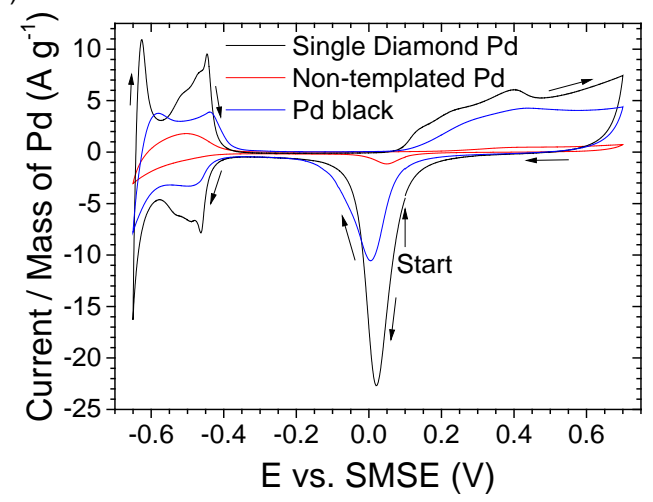

b)

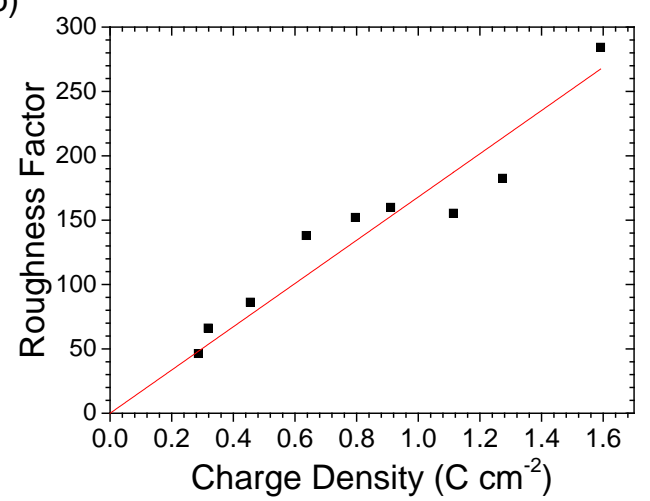

Figure 3. a) Cyclic voltammograms of single diamond Pd, Pd black and planar Pd recorded in $1 \mathrm{M} \mathrm{H}_{2} \mathrm{SO}_{4}$ at a scan rate of $20 \mathrm{mV} \mathrm{s}^{-1} \mathrm{vs}$ SMSE. Here all the second scans are compared so full oxygen stripping can be observed, with a maximum anodic potential of $+0.7 \mathrm{~V}$ vs. SMSE reached before reduction. For electrodeposited films this was calculated assuming 100\% faradaic efficiency. b) Roughness factor calculated for phytantriol templated Pd by dividing the calculated surface area from CVs in $1 \mathrm{M} \mathrm{H}_{2} \mathrm{SO}_{4}$ by the surface area of the substrate electrode. Charge density was calculated by dividing the charge passed whilst electrodepositing the single diamond Pd by the geometric surface area of the underlying electrode. The deposition substrate was a $3 \mathrm{~mm}$ diameter glassy carbon electrode.
Pd is shown to exhibit a peak current on the anodic scan for methanol electrooxidation of $1100 \mathrm{~A} \mathrm{~g}^{-1}$, which is $10 \times$ larger than $110 \mathrm{~A} \mathrm{~g}^{-1}$ shown by Pd black and over $50 \times$ larger than 21 $\mathrm{A} \mathrm{g}^{-1}$ shown by non-templated Pd. The peak current of $1100 \mathrm{~A}$ $\mathrm{g}^{-1}$ is larger than is seen in the literature for Pd electrooxidation of methanol. Yin et al. report a value of $210.5 \mathrm{~A} \mathrm{~g}^{-1}$ for $4.6 \mathrm{~nm}$ particle size Pd and up to $950.6 \mathrm{~A} \mathrm{~g}^{-1}$ with $70 \%$ Au incorporation. ${ }^{56}$ Yin et al. use a scan rate of $50 \mathrm{mV} \mathrm{s}^{-1}$ which makes the value for single diamond Pd more impressive (peak current $\alpha$ $\left.v^{0.5}\right)^{57,58} \cdot{ }^{56} 1100 \mathrm{~A} \mathrm{~g}^{-1}$ is also higher than $40 \mathrm{~A} \mathrm{~g}^{-1}\left(10 \mathrm{mV} \mathrm{s}^{-1}\right)$ reported for Pd nanoflowers, ${ }^{59}$ higher than $680 \mathrm{~A} \mathrm{~g}^{-1}\left(20 \mathrm{mV} \mathrm{s}^{-}\right.$ $\left.{ }^{1}\right)$ reported for $\mathrm{Ni}$ at $\mathrm{Pd}$ on multiwall carbon nanotubes, ${ }^{57}$ and higher than that even shown for Pt in acidic conditions, $600 \mathrm{~A}$ g-1 $\left(50 \mathrm{mV} \mathrm{s}^{-1}\right) .{ }^{60}$ These results are extremely encouraging, as they show single diamond Pd is very effective at the electrooxidation of methanol. The peak current is however strongly limited by mass transport for all three Pd catalysts in Figure 4a. To allow for a more reasonable comparison of the mass activities, currents were extracted at $-0.4 \mathrm{~V}$ vs. SCE. These currents can be seen in Figure 4g. Currents are also shown for surface specific activity where the current is normalized against surface area instead of mass. Figure S4 shows the cyclic voltammograms normalized against surface area. From Figure 4g single diamond Pd is seen to have the highest mass activity, but the surface specific activity is in line with that shown for Pd black. This means the increase in mass activity of single diamond Pd is due to only the increase in the surface area and not an increase in intrinsic surface specific activity of the Pd. These results still show however, that less mass of Pd is needed to perform the same level of methanol electrooxidation when in single diamond form compared to the Pd black form shown in this work. Chronoampometry (as seen in Figure 4b) shows a consistently higher rate of methanol electrooxidation per gram over time when using single diamond Pd compared to both non-templated Pd and Pd black. Figure S5 shows the chronoampometry normalized against surface area. These results are backed up with peak currents seen over 250 scans as seen in Figure S6, S7 and $\mathrm{S} 8$ in the supporting information.

From Figure 4c single Diamond Pd is shown to exhibit a peak ethanol electrooxidation current on the anodic scan of $116 \mathrm{~A} \mathrm{~g}$ 
${ }^{1}$, which is over $11 \times$ larger than $10 \mathrm{~A} \mathrm{~g}^{-1}$ shown by Pd black and non-templated Pd. The peak current of $116 \mathrm{~A} \mathrm{~g}^{-1}$ is similar to than seen for tetrahedral Pd (151 $\left.\mathrm{A} \mathrm{g}^{-1}\right)$ in the literature for
Pd electrooxidation of ethanol. ${ }^{30}$ These results are encouraging, as they show single diamond Pd is effective for the electrooxidation of ethanol. The peak current is however

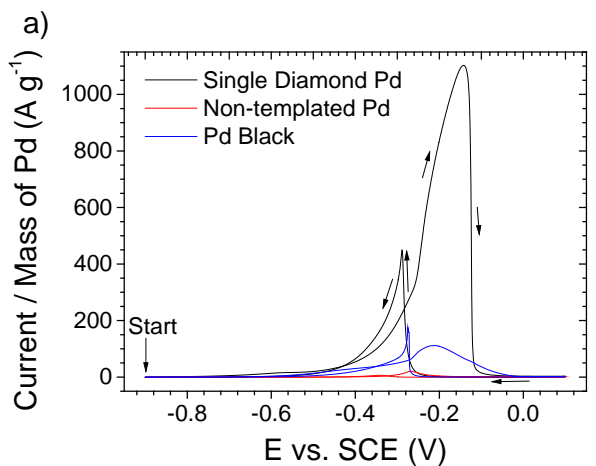

c)

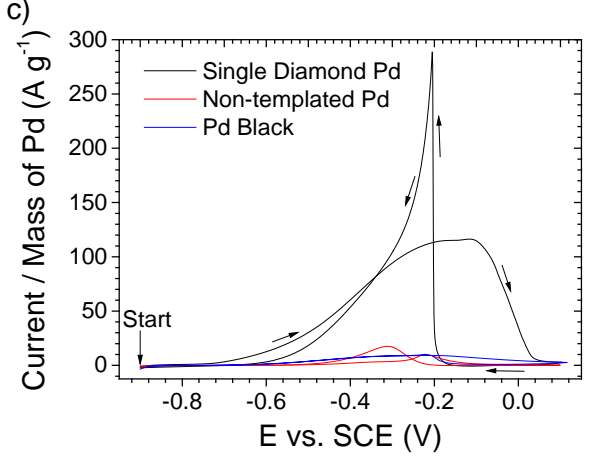

e)

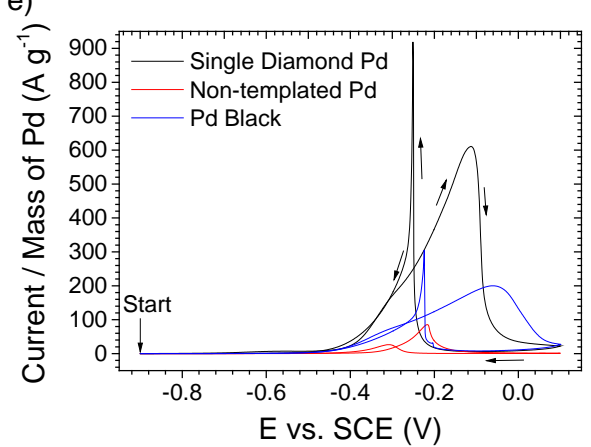

b)

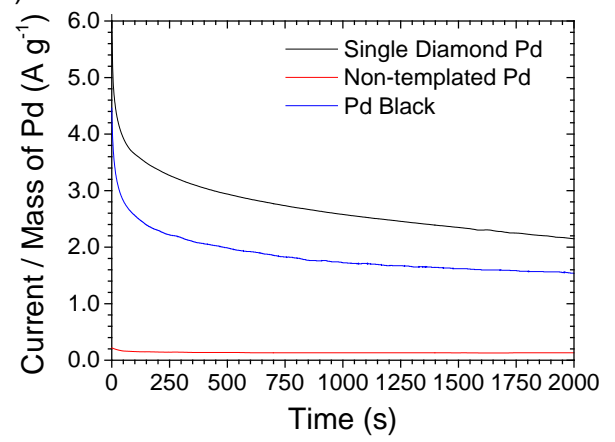

d)

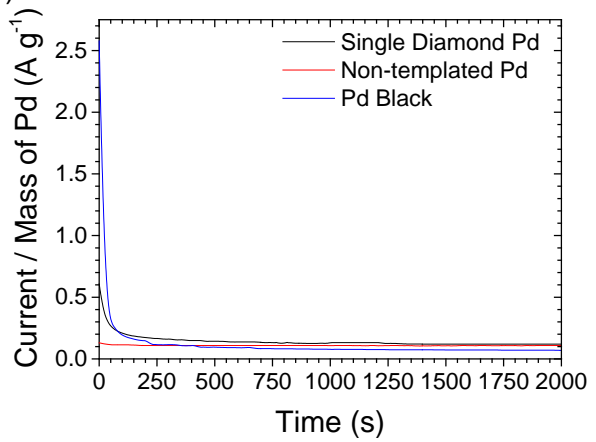

f)

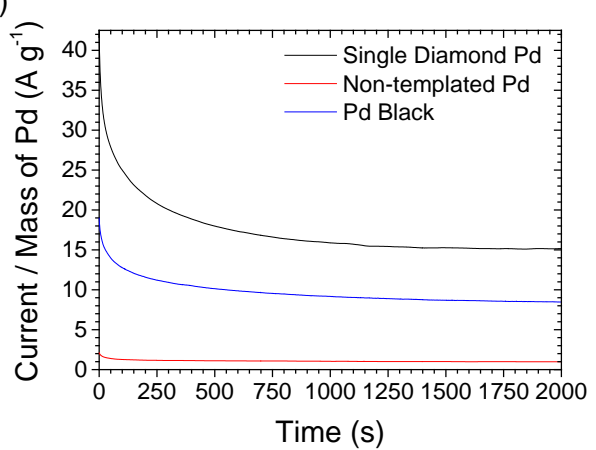

\begin{tabular}{|l|l|l|l|l|l|l|}
\hline g) & Methanol & Ethanol & \multicolumn{2}{l|}{ Glycerol } \\
\cline { 2 - 7 } & $\begin{array}{l}\text { Current / } \\
\text { Mass }\left(\mathrm{Ag}^{-1}\right)\end{array}$ & $\begin{array}{l}\text { Current / Sur- } \\
\text { face Area }(A \\
\left.\mathrm{Cm}^{-2}\right)\end{array}$ & $\begin{array}{l}\text { Current / } \\
\text { Mass }\left(\mathrm{Ag}^{-1}\right)\end{array}$ & $\begin{array}{l}\text { Current / Sur- } \\
\text { face Area }(A \\
\left.\mathrm{Cm}^{-2}\right)\end{array}$ & $\begin{array}{l}\text { Current / } \\
\text { Mass }\left(\mathrm{Ag}^{-1}\right)\end{array}$ & $\begin{array}{l}\text { Current / Sur- } \\
\text { face Area }(A \\
\left.\mathrm{cm}^{-2}\right)\end{array}$ \\
\hline $\begin{array}{l}\text { Single Dia- } \\
\text { mond Pd }\end{array}$ & 54.02774 & 1.75415 & 60.33125 & 1.95881 & 23.52166 & 0.76369 \\
\hline $\begin{array}{l}\text { Non-tem- } \\
\text { plated Pd }\end{array}$ & 0.93813 & 0.62183 & 2.06312 & 1.36753 & 1.69892 & 1.12612 \\
\hline Pd Black & 30.37123 & 1.70625 & 7.2012 & 0.40456 & 22.54928 & 1.26681 \\
\hline
\end{tabular}

Figure 4. a) Cyclic voltammetry of Single Diamond Pd, Pd black and planar Pd in 1 M methanol and 1 M KOH. b) Chronoampometry of Single Diamond Pd, Pd black and planar Pd in $1 \mathrm{M}$ methanol and $1 \mathrm{M} \mathrm{KOH}$, recorded at $+0.30 \mathrm{~V}$ vs. SCE. c) Cyclic voltammetry of Single Diamond Pd, Pd black and planar Pd in $0.1 \mathrm{M}$ ethanol and 0.1 M NaOH. d) Chronoampometry of Single Diamond Pd, Pd black and planar Pd in $0.1 \mathrm{M}$ ethanol and $0.1 \mathrm{M} \mathrm{NaOH}$, recorded at $+0.30 \mathrm{~V}$ vs. SCE. e) Cyclic voltammetry of Single Diamond Pd, Pd black and planar Pd in $1 \mathrm{M}$ glycerol and $1 \mathrm{M} \mathrm{KOH}$. f) Chronoampometry of Single Diamond Pd, Pd black and planar Pd in $1 \mathrm{M}$ glycerol and $1 \mathrm{M}$ KOH, recorded at $+0.30 \mathrm{~V}$ vs. SCE. g) Comparison of mass activities and specific activities for all 3 alcohols studied.

strongly limited by mass transport for all three Pd catalysts in Figure 4c. To allow for a more reasonable comparison of the mass activities, currents were extracted at $-0.4 \mathrm{~V}$ vs. SCE. These currents can be seen in Figure 4g. Currents are also shown for surface specific activity where the current is normalized against surface area instead of mass. Figure S9 shows the cyclic voltammograms normalized against surface area. From Figure 4g single diamond Pd is seen to have the highest mass activity, the surface specific activity is also seen to be the highest. This means the increase in mass activity of single diamond Pd is due 
to an increase in the surface area and an increase in intrinsic surface specific activity of the Pd. These results show that less Pd is needed to perform the same level of ethanol electrooxidation when in single diamond form compared to the Pd black form shown in this work. Chronoampometry (as seen in Figure 4d) shows a higher rate of ethanol electrooxidation per gram after $100 \mathrm{~s}$ when using single diamond Pd compared to both non-templated Pd and Pd black, showing single diamond Pd to be more durable than other Pd types tested here in the time range studied (2000 s). Figure S10 shows the chronoampometry normalised against surface area. These results are backed up with peak currents seen over 250 scans as seen in Figure S11, $\mathrm{S} 12$ and S13 in the supporting information.

From Figure 4e single Diamond Pd is shown to exhibit a peak current on the anodic scan of $612 \mathrm{~A} \mathrm{~g}^{-1}$, which is over $3 \times$ larger than $199 \mathrm{~A} \mathrm{~g}^{-1}$ shown by Pd black and over $7 \times$ larger than 85 $\mathrm{A} \mathrm{g}^{-1}$ shown by non-templated Pd. The peak current of $612 \mathrm{~A} \mathrm{~g}$ ${ }^{1}$ is larger than is seen in the literature for Pd electrooxidation of glycerol. Geraldes et al. report a value of $37 \mathrm{~A} \mathrm{~g}^{-1}$ for Pd on carbon with average particle sizes of around $4.0 \mathrm{~nm}$ particle size Pd and up to $54 \mathrm{~A} \mathrm{~g}^{-1}$ with $50 \%$ Au incorporation. ${ }^{61}$ Wang et al. use a scan rate of $50 \mathrm{mV} \mathrm{s}^{-1}$ and get a peak current of $257 \mathrm{~A} \mathrm{~g}^{-1}$ (peak current $\left.\alpha v^{0.5}\right)^{57,58},{ }^{58}$ using Pd on carbonised porous anodic alumina. This is still under $2 \times$ smaller, without factoring in the faster scan rate. These results are extremely encouraging, as they show single diamond Pd is very effective at the electrooxidation of glycerol. The peak current is however strongly limited by mass transport for all three Pd catalysts in Figure 4e. To allow for a more reasonable comparison of the mass activities, currents were extracted at $-0.4 \mathrm{~V}$ vs. SCE. These currents can be seen in Figure 4g. Currents are also shown for surface specific activity where the current is normalized against surface area instead of mass. Figure S14 shows the cyclic voltammograms normalized against surface area. From Figure 4g single diamond Pd is seen to have slightly higher mass activity than Pd black, but has lower surface specific activity than both Pd black and non-templated Pd. The opposite was true for methanol electrooxidation, where single diamond Pd showed the highest surface specific activity. A likely explanation for this contrast, is due to the increased steric hindrance present with the larger glycerol molecules, compared to the smaller methanol molecules. The single diamond architecture will provide greater hinderance when compared to either a planar film or isolated Pd black particles. This means the increase in mass activity of single diamond Pd is due to only the increase in the surface area and not an increase in intrinsic surface specific activity of the Pd. Chronoampometry (as seen in Figure 4f) shows a higher rate of glycerol electrooxidation per gram over time when using single diamond Pd compared to both non-templated Pd and Pd black, showing single diamond Pd to be more durable than other Pd types tested here in the time range studied (2000 s). Single diamond Pd is shown to have a current of $15 \mathrm{~A} \mathrm{~g}^{-1}$ after $2000 \mathrm{~s}$, which is higher than the $11 \mathrm{~A} \mathrm{~g}^{-1}$ shown by a 50:50 mixture of Pd and Au on $\mathrm{C}$ after 1800 s shown by Geraldes et al. (pure Pd on C produced $7 \mathrm{~A}$ g- 1 after $1800 \mathrm{~s}$ in the same paper). ${ }^{61}$ Figure S15 shows the chronoampometry normalized against surface area. These results are backed up with peak currents seen over 250 scans as seen in Figure S16, S17 and S18 in the supporting information.

\section{CONCLUSIONS}

In this study we demonstrate the fabrication of nanostructured Pd with a single diamond $\left(\mathrm{Q}_{227}\right)$ nanostructure. This was confirmed by SAXS (Figure 1). The presence of a $\mathrm{Q}_{227}$ nanostructure was further confirmed by STEM, FFT (Figure 2) and CVs in $1 \mathrm{M} \mathrm{H}_{2} \mathrm{SO}_{4}$ for surface area calculations (Figure 3).

Single diamond Pd was deposited with deposition charges ranging between $9 \mathrm{mC}$ and $50 \mathrm{mC}$. The surface area increased linearly with increasing deposition charge (Figure 3b), demonstrating a uniformly accessible nanostructure. From the gradient the specific electrochemical surface area to mass ratio and the surface area to volume ratio were estimated to be $30.8 \pm 0.4 \mathrm{~m}^{2}$ $\mathrm{g}^{-1}$ and $3.66( \pm 0.04) \times 106 \mathrm{~cm}^{2} \mathrm{~cm}^{-3}$ respectively.

Single diamond Pd was tested as a catalyst for the electrooxidation of alcohols to assess the potential use of single diamond Pd in alkaline fuel cells. For methanol electrooxidation (Figure 4a, 4b) single diamond Pd was shown to exhibit a peak current per mass on the anodic scan $10 \times$ larger than that seen for Pd black and over $50 \times$ larger than shown by non-templated Pd. The peak current of $1100 \mathrm{~A} \mathrm{~g}^{-1}$ is larger than is seen in the literature for $\mathrm{Pd},{ }^{56,57,59}$ and seen for $\mathrm{Pt}^{60}$ The peak current is however strongly limited by mass transport so currents in the surface kinetic limited regime were compared, where single diamond Pd was seen to have the highest mass activity, but the surface specific activity was in line with that shown for Pd black meaning the increase in mass activity is due to only an increase in the surface area. For ethanol electrooxidation (Figure 4c, 4d) single Diamond Pd was shown to exhibit a peak current per mass on the anodic scan $11 \times$ larger than was shown by Pd black and non-templated Pd. The peak current of $116 \mathrm{~A} \mathrm{~g}^{-1}$ is similar to than seen for Pd (151 $\left.\mathrm{A} \mathrm{g} \mathrm{g}^{-1}\right)$ in the literature. ${ }^{30}$ Currents in the surface kinetic limited regime were compared, where single diamond Pd is seen to have the highest mass activity and surface specific activity. Here the increase in mass activity of single diamond Pd appears to be due to an increase in the surface area and an increase in intrinsic surface specific activity of the Pd towards ethanol electrooxidation. For glycerol electrooxidation (Figure 4e, 4f) single Diamond Pd was shown to exhibit a peak current per mass on the anodic scan over $3 \times$ larger than was shown by Pd black and over $7 \times$ larger than was shown by nontemplated Pd. The peak current of $612 \mathrm{~A} \mathrm{~g}^{-1}$ is larger than is seen in the literature for Pd electrooxidation of glycerol. ${ }^{58,61}$ Currents in the surface kinetic limited regime were compared, where single diamond Pd was seen to have the highest mass activity, but the lowest surface specific activity meaning the increase in mass activity was due to only an increase in the surface area.

\section{ASSOCIATED CONTENT}

\section{Supporting Information.}

The Supporting Information is available free of charge on the ACS Publications website.

5 cycles for each Pd type for surface area characterization, all methanol, ethanol and glycerol CVs and chronoam-perometries with y axis plotted as current per unit area. Re-peated methanol, ethanol and glycerol electrooxidation scans for all types of Pd studied. CVs of Pd precursor electrolyte, with and without phytantriol template. XRD, SEM and EDX. 


\section{AUTHOR INFORMATION}

\section{Corresponding Author}

*iris@soton.ac.uk

\section{Author Contributions}

Matthew Burton and Iris Nandhakumar contributed equally to the writing of the manuscript. Anand Selvam acquired some of the cyclic voltammograms. Adam Squires provided the Matlab simulations for the HAAD-STEM images. Jake-Lawrie Ashton electrodeposited Pd nanostructures onto TEM grids and Nick Terrill advised on SAXS data acquisition and analy-sis.

\section{Funding Sources}

Any funds used to support the research of the manuscript should be placed here (per journal style).

Notes

Any additional relevant notes should be placed here.

\section{ACKNOWLEDGMENT}

We are grateful to Diamond light source for allocation of beam times SM14925 and SI10330 on beam line I22 and EM16964-1 and EM16964-2 on ePSIc. The authors acknowledge the financial support of equipment grant (EP/K00509X/1) for the SmartLab.

\section{REFERENCES}

(1) Hübert, T.; Boon-Brett, L.; Black, G.; Banach, U. Hydrogen Sensors - A Review. Sensors Actuators B Chem. 2011, 157 (2), 329-352.

(2) Hübert, T.; Boon-Brett, L.; Palmisano, V.; Bader, M. A. Developments in Gas Sensor Technology for Hydrogen Safety. Int. J. Hydrogen Energy 2014, 39 (35), 20474-20483.

(3) Meng, L.; Jin, J.; Yang, G.; Lu, T.; Zhang, H.; Cai, C. Nonenzymatic Electrochemical Detection of Glucose Based on Palladium-Single-Walled Carbon Nanotube Hybrid Nanostructures. Anal. Chem. 2009, 81 (17), 7271-7280. Łukaszewski, M.; Hubkowska, K.; Koss, U.; Czerwiński, A. Characteristic of Hydrogen-Saturated Pd-Based Alloys for the Application in Electrochemical Capacitors. J. Solid State Electrochem. 2012, 16 (7), 2533-2539.

(5) Lukaszewski, M.; Żurowski, A.; Czerwiński, A. Hydrogen in Thin Pd-Based Layers Deposited on Reticulated Vitreous Carbon-A New System for Electrochemical Capacitors. J. Power Sources 2008, 185 (2), 1598-1604.

(6) Chen, A.; Ostrom, C. Palladium-Based Nanomaterials: Synthesis and Electrochemical Applications. Chem. Rev. 2015, 115 (21), 11999-12044.

(7) Savadogo, O.; Lee, K.; Oishi, K.; Mitsushima, S.; Kamiya, N.; Ota, K.-I. New Palladium Alloys Catalyst for the Oxygen Reduction Reaction in an Acid Medium. Electrochem. commun. 2004, 6 (2), 105-109.

(8) Gu, L.; Luo, N.; Miley, G. H. Cathode Electrocatalyst Selection and Deposition for a Direct Borohydride/Hydrogen Peroxide Fuel Cell. J. Power Sources 2007, 173 (1), 77-85.

(9) Yoon, S. R.; Hwang, G. H.; Cho, W. I.; Oh, I.-H.; Hong, S.-A.; Ha, H. Y. Modification of Polymer Electrolyte Membranes for DMFCs Using Pd Films Formed by Sputtering. J. Power Sources 2002, 106 (1), 215-223.

(10) Mougenot, M.; Caillard, A.; Brault, P.; Baranton, S.; Coutanceau, C. High Performance Plasma Sputtered PdPt Fuel Cell Electrodes with Ultra Low Loading. Int. J. Hydrogen Energy 2011, 36 (14), 8429-8434.

(11) Ocola, L. E.; Rue, C.; Maas, D. High-Resolution Direct-Write Patterning Using Focused Ion Beams. MRS Bull. 2014, 39 (04), 336-341.

(12) Spencer, J. A.; Rosenberg, S. G.; Barclay, M.; Wu, Y.-C.; McElwee-White, L.; Howard Fairbrother, D. Understanding the Electron-Stimulated Surface Reactions of Organometallic Complexes to Enable Design of Precursors for Electron BeamInduced Deposition. Appl. Phys. A 2014, 117 (4), 1631-1644.

Kochemirovsky, V. A.; Menchikov, L. G.; Tumkin, I. I.;

$$
\text { (12), } 1502 .
$$

Safavi, A.; Maleki, N.; Tajabadi, F.; Farjami, E. High Electrocatalytic Effect of Palladium Nanoparticle Arrays Electrodeposited on Carbon Ionic Liquid Electrode. Electrochem.

Logunov, L. S.; Safonov, S. V. Laser-Induced Chemical Liquid Phase Deposition of Copper from Aqueous Solutions without Reducing Agents. Quantum Electron. 2012, 42 (8), 693-695.

Cristoforetti, G.; Pitzalis, E.; Spiniello, R.; Ishak, R.; MunizMiranda, M. Production of Palladium Nanoparticles by Pulsed Laser Ablation in Water and Their Characterization. J. Phys. Chem. C 2011, 115 (12), 5073-5083.

Guo, P.; Wei, Z.; Ye, W.; Qin, W.; Wang, Q.; Guo, X.; Lu, C.; Zhao, X. S. Preparation and Characterization of Nanostructured Pd with High Electrocatalytic Activity. Colloids Surfaces A Physicochem. Eng. Asp. 2012, 395, 75-81.

Kuai, L.; Yu, X.; Wang, S.; Sang, Y.; Geng, B. Au-Pd Alloy and Core-Shell Nanostructures: One-Pot Coreduction Preparation, Formation Mechanism, and Electrochemical Properties. Langmuir 2012, 28 (18), 7168-7173.

Yi, Q.; Niu, F.; Sun, L. Fabrication of Novel Porous Pd Particles and Their Electroactivity towards Ethanol Oxidation in Alkaline Media. Fuel 2011, 90 (8), 2617-2623.

, M. H.; Huang, T.; Liu, P.; Zhang, J.; Sasaki, K.; Vukmirovic, M. B.; Adzic, R. R. Palladium Monolayer and Palladium Alloy Electrocatalysts for Oxygen Reduction $†$. Langmuir 2006, 22 (25), 10409-10415.

Lu, Y.; Chen, W. Nanoneedle-Covered Pd-Ag Nanotubes: High Electrocatalytic Activity for Formic Acid Oxidation. J. Phys. Chem. C 2010, 114 (49), 21190-21200.

Ojani, R.; Abkar, Z.; Hasheminejad, E.; Raoof, J.-B. Rapid Fabrication of $\mathrm{Cu} / \mathrm{Pd}$ Nano/Micro-Particles Porous-Structured Catalyst Using Hydrogen Bubbles Dynamic Template and Their Enhanced Catalytic Performance for Formic Acid Electrooxidation. Int. J. Hydrogen Energy 2014, 39 (15), 77887797.

Zarsen, R.; Ha, S.; Zakzeski, J.; Masel, R. I. Unusually Active Palladium-Based Catalysts for the Electrooxidation of Formic Acid. J. Power Sources 2006, 157 (1), 78-84.

Xu, C.; Cheng, L.; Shen, P.; Liu, Y. Methanol and Ethanol Electrooxidation on Pt and Pd Supported on Carbon Microspheres in Alkaline Media. Electrochem. commun. 2007, 9 (5), 997-1001.

De Vogelaere, M.; Sommer, V.; Springborn, H.; MichelsenMohammadein, U. High-Speed Plating for Electronic Applications. Electrochim. Acta 2001, 47 (1-2), 109-116.

Chen, H.; Wei, Z.; Zheng, X.; Yang, S. A Scalable Electrodeposition Route to the Low-Cost, Versatile and Controllable Fabrication of Perovskite Solar Cells. Nano Energy 2015, 15, 216-226.

Lee, C.-H.; Wang, S.-C.; Yuan, C.-J.; Wen, M.-F.; Chang, K.-S. Comparison of Amperometric Biosensors Fabricated by Palladium Sputtering, Palladium Electrodeposition and Nafion/Carbon Nanotube Casting on Screen-Printed Carbon Electrodes. Biosens. Bioelectron. 2007, 22 (6), 877-884.

Arenz, M.; Stamenkovic, V.; Schmidt, T. J.; Wandelt, K.; Ross, P. N.; Markovic, N. M. The Electro-Oxidation of Formic Acid on Phys. 2003, 5 (19), 4242-4251.

Shi, Q.; Diao, G. The Electrocatalytical Reduction of MNitrophenol on Palladium Nanoparticles Modified Glassy Carbon Electrodes. Electrochim. Acta 2011, 58, 399-405.

Jiang, Y.; Lu, Y.; Li, F.; Wu, T.; Niu, L.; Chen, W. Facile Electrochemical Codeposition of "Clean" Graphene-Pd Nanocomposite as an Anode Catalyst for Formic Acid Electrooxidation. Electrochem. commun. 2012, 19, 21-24.

Zhao, Y.; Qin, S.-J.; Li, Y.; Deng, F.-X.; Liu, Y.-Q.; Pan, G.-B. Electrodeposition of Dendritic Pd Nanoarchitectures on NGaN(0001): Nucleation and Electrocatalysis for Direct Formic Acid Fuel Cells. Electrochim. Acta 2014, 145, 148-153. Electrodeposition of Tetrahexahedral Pd Nanocrystals with HighIndex Facets and High Catalytic Activity for Ethanol Electrooxidation. J. Am. Chem. Soc. 2010, 132 (22), 7580-7581. Tian, N.; Zhou, Z.-Y.; Sun, S.-G. Electrochemical Preparation of 
commun. 2007, 9 (8), 1963-1968.

Liu, J.; Ye, J.; Xu, C.; Jiang, S. P.; Tong, Y. Kinetics of Ethanol Electrooxidation at Pd Electrodeposited on Ti. Electrochem. commun. 2007, 9 (9), 2334-2339.

(34) Mustain, W. E.; Prakash, J. Kinetics and Mechanism for the Oxygen Reduction Reaction on Polycrystalline Cobalt-palladium Electrocatalysts in Acid Media. J. Power Sources 2007, 170 (1), 28-37.

(35) Lim, S. H.; Wei, J.; Lin, J.; Li, Q.; KuaYou, J. A Glucose Biosensor Based on Electrodeposition of Palladium Nanoparticles and Glucose Oxidase onto Nafion-Solubilized Carbon Nanotube Electrode. Biosens. Bioelectron. 2005, 20 (11), 2341-2346.

(36) Łukaszewski, M.; Czerwiński, A. Selected Electrochemical Properties of Pd-Au Alloys: Hydrogen Absorption and Surface Oxidation. J. Solid State Electrochem. 2008, 12 (12), 1589-1598.

(37) Sarto, F.; Castagna, E.; De Francesco, M.; Dikonimos, T. M. Giorgi, L.; Lecci, S.; Sansovini, M.; Violante, V. Morphology and Electrochemical Properties of Pd-Based Catalysts Deposited by Different Thin-Film Techniques. Int. J. Hydrogen Energy 2014, 39 (27), 14701-14711.

(38) Renjith, A.; Lakshminarayanan, V. One Step Preparation of 'Ready to Use' Au@Pd Nanoparticle Modified Surface Using Deep Eutectic Solvents and a Study of Its Electrocatalytic Properties in Methanol Oxidation Reaction. J. Mater. Chem. A 2015, 3 (6), 3019-3028.

(39) Choi, S.; Jeong, H.; Choi, K.; Song, J. Y.; Kim, J. Electrodeposition of Triangular Pd Rod Nanostructures and Their Electrocatalytic and SERS Activities. ACS Appl. Mater. Interfaces 2014, 6 (4), 3002-3007.

(40) Lu, X.; Luo, F.; Song, H.; Liao, S.; Li, H. Pulse Electrodeposition to Prepare Core-shell Structured AuPt@Pd/C Catalyst for Formic Acid Fuel Cell Application. J. Power Sources 2014, 246, 659666.

(41) Wang, H.; Xu, C.; Cheng, F.; Jiang, S. Pd Nanowire Arrays as Electrocatalysts for Ethanol Electrooxidation. Electrochem. commun. 2007, 9 (5), 1212-1216.

(42) Cui, C.-H.; Yu, J.-W.; Li, H.-H.; Gao, M.-R.; Liang, H.-W.; Yu, S.-H. Remarkable Enhancement of Electrocatalytic Activity by Tuning the Interface of $\mathrm{Pd}-\mathrm{Au}$ Bimetallic Nanoparticle Tubes. ACS Nano 2011, 5 (5), 4211-4218.

(43) Cheng, F.; Wang, H.; Sun, Z.; Ning, M.; Cai, Z.; Zhang, M. Electrodeposited Fabrication of Highly Ordered Pd Nanowire Arrays for Alcohol Electrooxidation. Electrochem. commun. 2008, 10 (5), 798-801.

(44) Liu, L.; Yoo, S.-H.; Lee, S. A.; Park, S. Wet-Chemical Synthesis of Palladium Nanosprings. Nano Lett. 2011, 11 (9), 3979-3982.

(45) Bai, H.; Han, M.; Du, Y.; Bao, J.; Dai, Z. Facile Synthesis of Porous Tubular Palladium Nanostructures and Their Application in a Nonenzymatic Glucose Sensor. Chem. Commun. 2010, 46 (10), 1739.

(46) Bartlett, P. N.; Gollas, B.; Guerin, S.; Marwan, J. The Preparation and Characterisation of H1-e Palladium Films with a Regular Hexagonal Nanostructure Formed by Electrochemical Deposition from Lyotropic Liquid Crystalline Phases. Phys. Chem. Chem. Phys. 2002, 4 (15), 3835-3842.
System. Langmuir 2003, 19 (23), 9562-9565.

Burton, M. R.; Lei, C.; Staniec, P. A.; Terrill, N. J.; Squires, A. M.; White, N. M.; Nandhakumar, I. S. 3D Semiconducting Nanostructures via Inverse Lipid Cubic Phases. Sci. Rep. 2017, 7 (1), 6405.

(49) Burton, M. R.; Richardson, S. J.; Staniec, P. A.; Terrill, N. J.; Elliott, J. M.; Squires, A. M.; White, N. M.; Nandhakumar, I. S. A Novel Route to Nanostructured Bismuth Telluride Films by Electrodeposition. Electrochem. commun. 2017, 76, 71-74.

(50) Richardson, S. J.; Burton, M. R.; Staniec, P. A.; Nandhakumar, I. S.; Terrill, N. J.; Elliott, J. M.; Squires, A. M. Aligned Platinum Nanowire Networks from Surface-Oriented Lipid Cubic Phase Templates. Nanoscale 2016, 8 (5), 2850-2856.

(51) Martinez, U.; Serov, A.; Padilla, M.; Atanassov, P. Mechanistic Insight into Oxide-Promoted Palladium Catalysts for the ElectroOxidation of Ethanol. ChemSusChem 2014, 7 (8), 2351-2357.

(52) Wang, W.; Yang, Y.; Liu, Y.; Zhang, Z.; Dong, W.; Lei, Z. Hybrid NiCoO x Adjacent to Pd Nanoparticles as a Synergistic Electrocatalyst for Ethanol Oxidation. J. Power Sources 2015, 273, 631-637.

(53) Akbar, S.; Elliott, J. M.; Rittman, M.; Squires, A. M. Facile Production of Ordered 3D Platinum Nanowire Networks with "Single Diamond” Bicontinuous Cubic Morphology. Adv. Mater. 2013, 25 (8), 1160-1164.

(54) Richardson, S. J.; Burton, M. R.; Luo, X.; Staniec, P. A.; Nandhakumar, I. S.; Terrill, N. J.; Elliott, J. M.; Squires, A. M. Watching Mesoporous Metal Films Grow during Templated Electrodeposition with in Situ SAXS. Nanoscale 2017, 9 (29), 10227-10232.

(55) Rand, D. A. J.; Woods, R. The Nature of Adsorbed Oxygen on Rhodium, Palladium and Gold Electrodes. J. Electroanal. Chem. Interfacial Electrochem. 1971, 31 (1), 29-38.

(56) Yin, Z.; Chi, M.; Zhu, Q.; Ma, D.; Sun, J.; Bao, X. Supported Bimetallic PdAu Nanoparticles with Superior Electrocatalytic Activity towards Methanol Oxidation. J. Mater. Chem. A 2013, 1 (32), 9157-9163.

(57) Zhao, Y.; Yang, X.; Tian, J.; Wang, F.; Zhan, L. Methanol Electro-Oxidation on Ni@Pd Core-Shell Nanoparticles Supported on Multi-Walled Carbon Nanotubes in Alkaline Media. Int. J. Hydrogen Energy 2010, 35 (8), 3249-3257.

(58) Wang, Z.; Hu, F.; Shen, P. K. Carbonized Porous Anodic Alumina as Electrocatalyst Support for Alcohol Oxidation. Electrochem. commun. 2006, 8 (11), 1764-1768.

(59) Yin, Z.; Zheng, H.; Ma, D.; Bao, X. Porous Palladium Nanoflowers That Have Enhanced Methanol Electro-Oxidation Activity. J. Phys. Chem. C 2009, 113 (3), 1001-1005.

(60) Chung, D. Y.; Lee, K.-J.; Sung, Y.-E. Methanol ElectroOxidation on the Pt Surface: Revisiting the Cyclic Voltammetry Interpretation. J. Phys. Chem. C 2016, 120 (17), 9028-9035.

(61) Geraldes, A. N.; Silva, D. F.; Silva, J. C. M.; Souza, R. F. B.; Spinacé, E. V.; Oliveira Neto, A.; Linardi, M.; Santos, M. C. Glycerol Electrooxidation in Alkaline Medium Using $\mathrm{Pd} / \mathrm{C}, \mathrm{Au} / \mathrm{C}$ and $\mathrm{PdAu} / \mathrm{C}$ Electrocatalysts Prepared by Electron Beam Irradiation. J. Braz. Chem. Soc. 2014, 25 (5), 831-840. 
Graphical Abstract

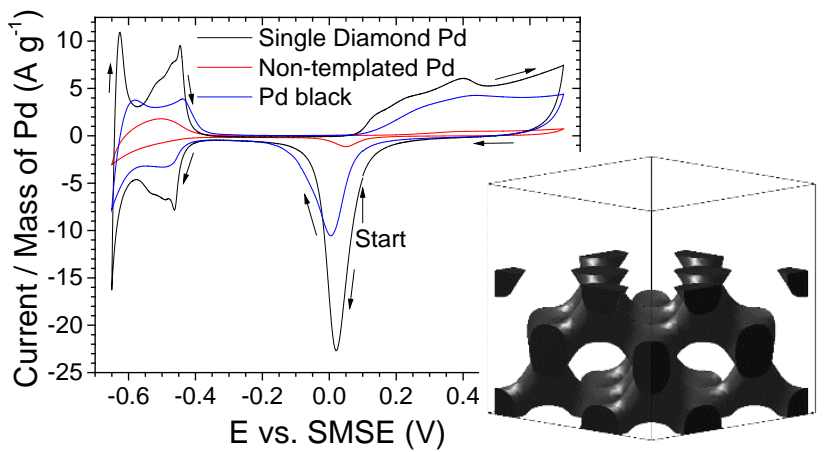

